\title{
Time delay in two-electron photodetachment and tests of fundamental threshold laws
}

\author{
Anatoli S. Kheifets $\odot$ \\ Research School of Physics and Engineering, The Australian National University, Canberra ACT 2600, Australia \\ I. Bray $\odot$ \\ Curtin Institute for Computation and Department of Physics, Curtin University, Perth WA 6845, Australia
}

(Received 16 March 2021; accepted 14 September 2021; published 7 October 2021)

\begin{abstract}
The photoelectron group delay, also known as the Wigner time delay (WTD), is examined in photodetachment of the $\mathrm{H}^{-}$and $\mathrm{Li}^{-}$ions near their lowest inelastic and the double detachment thresholds. These threshold processes promoting the remaining bound electron to an excited state or ejecting it to the continuum involve two active electrons and are termed collectively two-electron photodetachment. The opening of an inelastic channel is governed by the Wigner threshold law which affects the WTD very differently in $\mathrm{H}^{-}$and $\mathrm{Li}^{-}$. At the same time, the Wannier threshold behavior of the WTD in double detachment is similar in both targets. Experimental verification of these theoretical predictions will subject the Wigner and Wannier threshold laws to a set of stringent tests in the time domain.
\end{abstract}

DOI: 10.1103/PhysRevResearch.3.043017

\section{INTRODUCTION}

Threshold phenomena play a special role in physics by revealing the essential link between weakly bound and fragmented systems [1]. A small excess energy and a large de Broglie wavelength of the particles escaping near the threshold allow for an analytical treatment of the problem. The threshold theories by Wigner [2] and Wannier [3] present examples of such a treatment. The Wigner and Wannier threshold laws play fundamental roles in atomic, molecular, and nuclear physics. These laws are being constantly applied to new classes of collision processes [4]; their validity is challenged [5] and ultimately reaffirmed [6]. Up to now, these tests of the fundamental Wigner and Wannier laws have been conducted in the energy domain. New techniques of attosecond chronoscopy [7] allow one to extend these tests to the time domain.

In the present work, we analyze the Wigner time delay (WTD) near the lowest excitation and the double detachment thresholds of the negative ions. The WTD was introduced into particle scattering theory by Eisenbud [8], Wigner [9], and Smith [10]. More recently, this concept was adopted in photoemission [11,12] and became the basis of experimental attosecond chronoscopy [7]. The WTD is a sensitive tool of photoemission especially under conditions where the photoelectron phase is strongly affected by resonances [13-16], inelastic channel openings [17], and intershell correlation [12].

Published by the American Physical Society under the terms of the Creative Commons Attribution 4.0 International license. Further distribution of this work must maintain attribution to the author(s) and the published article's title, journal citation, and DOI.
Photodetachment of negative atomic ions is influenced by many-electron correlation profusely. The sequence of negative ions with a closed $n s^{2}$ valence shell, from $\mathrm{H}^{-}$to $\mathrm{Cs}^{-}$, demonstrates the strength of this correlation very vividly. The presence of numerous resonances, Wigner cusps, and the sheer binding of these systems is underpinned by strong two-electron correlation [18-23]. We exploit these effects and study the WTD in the negative ions with two active electrons under conditions where it is affected most strongly by interelectron correlation. For our case studies, we select the $\mathrm{H}^{-}$ and $\mathrm{Li}^{-}$ions which display a very different manifestation of the Wigner law near their respective lowest excitation thresholds. While a short range potential of negative ions supports a limited number of bound states, the remaining neutral species have an infinite discrete spectrum which can be explored in photodetachment accompanied with excitation. In addition, we consider double detachment of the $\mathrm{H}^{-}$and $\mathrm{Li}^{-}$ions promoting both target electrons into the continuum and exploring the implications of the Wannier threshold law for the WTD.

\section{THEORETICAL MODEL}

Our theoretical model is based on the multiconfiguration Hartree-Fock (MCHF) expansion for the two-electron ground state and the convergent close-coupling (CCC) expansion of the final state with one or two electrons in the continuum. This approach has been tested thoroughly for single and double ionization of the helium isoelectronic sequence of ions including $\mathrm{H}^{-}$[24] and the alkaline earth metal atoms [25]. Expansion of this model to $\mathrm{Li}^{-}$and beyond is straightforward.

We make a configuration-interaction expansion of the twoelectron valence shell

$$
\Psi_{0}\left(\boldsymbol{r}_{1}, \boldsymbol{r}_{2}\right)=\sum_{l=0}^{l_{\max }} \sum_{m=n}^{n_{\max }} C_{m l}\left|\psi_{m l}\left(\boldsymbol{r}_{1}\right) \psi_{m l}\left(\boldsymbol{r}_{2}\right):{ }^{1} S\right\rangle .
$$


TABLE I. MCHF bases of two-electron bound systems: the He atom and the $\mathrm{H}^{-}$and $\mathrm{Li}^{-}$ions.

\begin{tabular}{lccc}
\hline \hline $\begin{array}{l}\text { Atom } \\
\text { expansion }\end{array}$ & $\begin{array}{c}\mathrm{He} \\
\mathrm{MCHF15}\end{array}$ & $\begin{array}{c}\mathrm{H} \\
\mathrm{MCHF9}\end{array}$ & $\begin{array}{c}\mathrm{Li}^{-} \\
\mathrm{MCHF} 8\end{array}$ \\
\hline$C_{m l} 1 s^{2}$ & 0.996 & 0.974 & \\
$2 s^{2}$ & -0.059 & -0.191 & 0.936 \\
$2 p^{2}$ & 0.059 & 0.111 & 0.335 \\
$R_{n}^{\infty}(\%) n=1$ & 94.541 & 63.409 & \\
2 & 4.469 & 35.735 & 78.521 \\
3 & 0.564 & 0.407 & 20.549 \\
$I_{p}(\mathrm{eV})$ & 24.579 & 0.738 & 0.651 \\
Expt. & 24.587 & 0.754 & 0.618 \\
Refs. & {$[27]$} & {$[28]$} & {$[29]$} \\
\hline \hline
\end{tabular}

The MCHF orbitals $\psi_{m l}(\boldsymbol{r})$ are found in the frozen $1 s^{2}$ core in the case of $\mathrm{Li}^{-}(n=2)$, while this core is absent in the case of $\mathrm{H}^{-}(n=1)$. Only diagonal $m l^{2}$ terms are included in expansion (1) as is always the case for the closed-shell MCHF ground state.

The coefficients $C_{m l}$ in the MCHF expansion (1) are found by using the multiconfiguration Dirac-Fock computer code [26]. The number of terms in the MCHF expansion is increased until we are satisfied with the stability of the ground state energy and the asymptotic photoionization ratios taken in the limit of infinite photon energy $R_{n}^{\infty}=\sigma_{n} /\left.\sigma^{+}\right|_{\omega \rightarrow \infty}$, where $\sigma_{n}$ is a single photoionization cross section corresponding to an $n$ excited state of the parentic species.

A few of the largest MCHF coefficients $C_{m l}$ and the asymptotic ratios $R_{n}^{\infty}$ are listed in Table I. We also display the ionization potential $I_{p}$. For comparison, we also show the corresponding values for the ground state He. As compared to $\mathrm{He}$, the $\mathrm{H}^{-}$and $\mathrm{Li}^{-}$ions are much stronger correlated with the weights of the virtual excitations in the ground state exceeding that of He three to four times. Accordingly, the singly charged $\mathrm{He}^{+}$ion remains predominantly in its ground state, while the $n=2$ and higher excitations are barely noticeable. After photodetachment of negative ions, the neutral atom in the final state has a very significant probability to be excited.

The cross section of a two-electron transition, measured as a function of the photon energy $\omega$ and corresponding to a particular state $j$ of the remaining bound electron, is given by [30]

$$
\sigma_{j}(\omega)=\frac{4 \pi^{2}}{\omega c} \bigcup_{m_{j}} d^{3} k\left|\left\langle\Psi_{j}^{(-)}(\boldsymbol{k})|\mathcal{D}| \Psi_{0}\right\rangle\right|^{2} \delta\left(\omega-E+E_{0}\right) .
$$

Here $c \simeq 137$ is the speed of light in atomic units, while other fundamental constants are set to $e=m=\hbar=1$.

The dipole matrix element entering Eq. (2) is calculated by integration of the bare two-electron dipole transition matrix with the half off-shell $T$ matrix [31]

$$
\begin{aligned}
\left\langle\Psi_{j}^{(-)}(\boldsymbol{k})|\mathcal{D}| \Psi_{0}\right\rangle= & \left\langle\boldsymbol{k}^{(-)} j|\mathcal{D}| \Psi_{0}\right\rangle+\sum_{i} \bigvee d^{3} \kappa \\
& \times \frac{\left\langle\boldsymbol{k}^{(-)} j|T| i \boldsymbol{\kappa}^{(+)}\right\rangle\left\langle\boldsymbol{\kappa}^{(+)} i|\mathcal{D}| \Psi_{0}\right\rangle}{E-\varepsilon_{\boldsymbol{\kappa}}-\epsilon_{i}+i 0} .
\end{aligned}
$$

Here the channel wave function $\left\langle\boldsymbol{k}^{(-)} j\right|$ is the product of a one-electron target orbital $\varphi_{j}$ with energy $\epsilon_{j}$ and a distorted outgoing wave $\chi^{(-)}(\boldsymbol{k})$ with energy $\varepsilon_{\boldsymbol{k}}$. For negative ions, the target orbital is generated with the asymptotic charge being one and the asymptotic charge seen by the distorted wave is zero. The contribution from the final channels $\left\langle\boldsymbol{k}_{b}^{(-)} j\right|$ is separated into single and double ionization according to the energy $\epsilon_{j}$, which is positive for the double ionized channels and negative for the singly ionized channels. The number of the target states is increased until satisfactory convergence is achieved. In practice, our calculations were performed with at least $20-\ell$ target states, where $0 \leqslant \ell \leqslant \ell_{\max }$ is the angular momentum of the target orbital and $\ell_{\max }=3$. For near threshold double detachment calculations, $\ell_{\max }=6$.

The phase of the transition amplitude

$$
\delta_{j}(\boldsymbol{k})=\arg \left\langle\Psi_{j}^{(-)}(\boldsymbol{k})|\mathcal{D}| \Psi_{0}\right\rangle, \quad \tau_{j}(\boldsymbol{k})=\partial \delta_{j}(\boldsymbol{k}) / \partial E
$$

is used to calculate the WTD $\tau_{j}(\boldsymbol{k})$ in the given emission direction as the phase derivative with respect to the photoelectron energy $E=k^{2} / 2$. If only one channel makes a dominant contribution into the transition amplitude (3), then the WTD becomes the energy derivative of the scattering phase in this particular channel $\eta_{j}(k)$. As such, it is independent on the photoelectron emission direction.

\section{NUMERICAL RESULTS}

\section{A. Photodetachment with excitation}

The photodetachment cross section (2) as well as the phase and the WTD (4) of $\mathrm{H}^{-}$near the $n=2$ threshold are presented in Fig. 1. The top panel displays the partial photodetachment cross sections in various channels leaving the $\mathrm{H}$ atom in the ground $1 s$ and excited $2 s$ or $2 p$ states. The total cross section is compared with the experiment [32]. The sharp resonance below the $n=2$ threshold is due to autodetachment of a two-electron bound state into the ground state of the $\mathrm{H}$ atom (Feshbach resonance). The $\mathrm{H}^{-}$ion supports several similar resonances converging to higher $n$ thresholds [33]. When a resonance decays into a single continuum, the Fano parametrization of the amplitude and the cross section takes the form [34]

$$
f(\epsilon)=f_{0} \frac{q+\epsilon}{i+\epsilon}, \quad \sigma(\epsilon)=\sigma_{0} \frac{(q+\epsilon)^{2}}{1+\epsilon^{2}} .
$$

Here $\epsilon=\left(E-E_{0}\right) /(\Gamma / 2)$ is the dimensionless energy counted from the center of the resonance and measured in its half width and $q$ is the so-called profile index. The nonresonant amplitude $f_{0}$ changes little over the width of the resonance. In such a case, the phase and the WTD becomes respectively

$$
\arg f(\epsilon)=-\arctan \frac{1}{\epsilon}, \quad \tau(\epsilon)=\frac{1}{1+\epsilon^{2}} \frac{2}{\Gamma}, \quad \tau_{0}=2 / \Gamma .
$$

The peak WTD at the center of the resonance $\tau_{0}$ corresponds to the dwelling time the electron spends when it is captured temporarily into the resonance. Note that the WTD (6) is always positive. This case is realized for the narrow resonance centered at $\omega=10.927 \mathrm{eV}$, which decays into the $1 s \rightarrow E p$ 

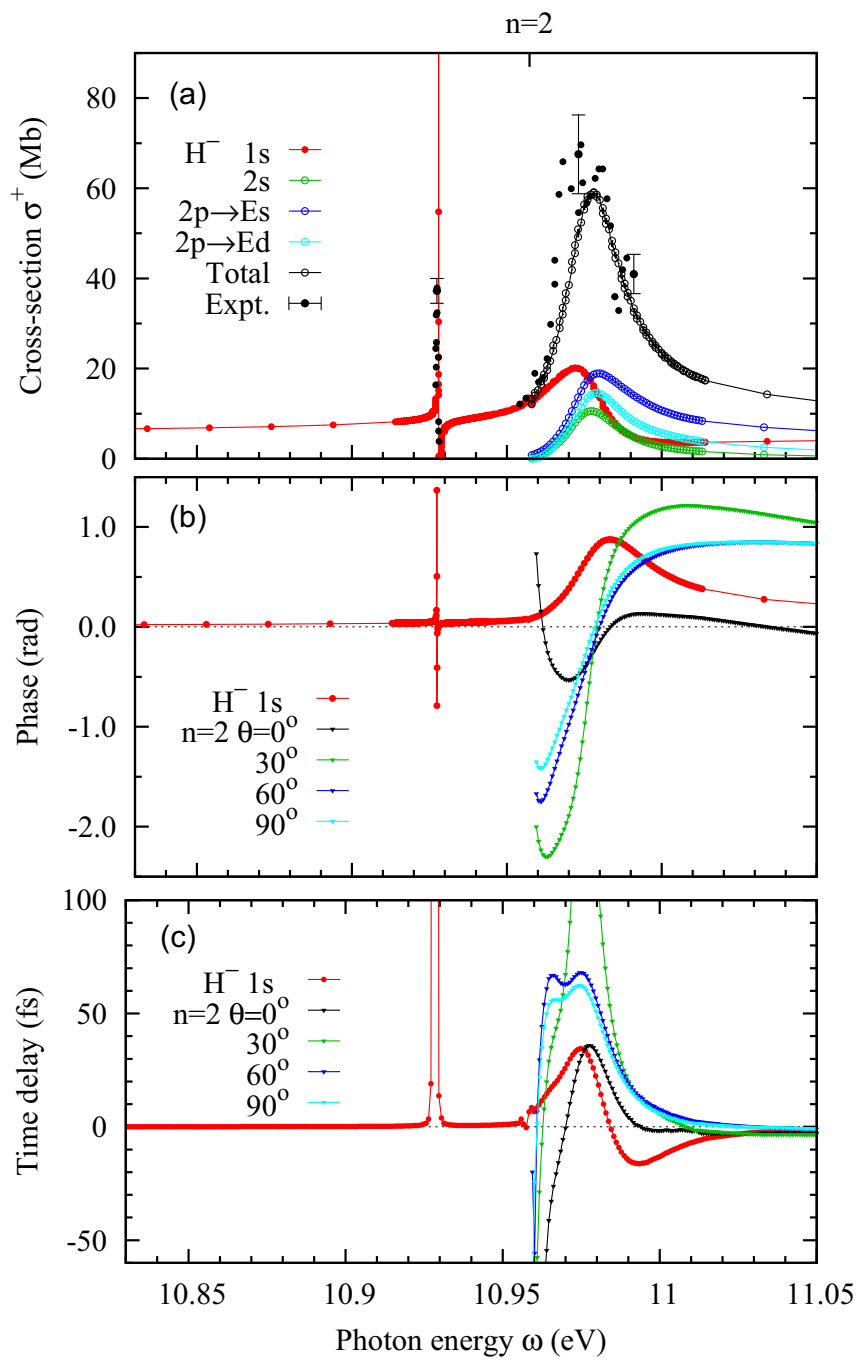

FIG. 1. (a) Cross section (2), (b) the phase of the transition amplitude, and (c) the WTD (4) of $\mathrm{H}^{-}$in various photodetachment channels near the $n=2$ threshold. The experiment [32] is displayed in the top panel.

continuum. Here fitting the cross section with the squared amplitude (5) returns $\Gamma \simeq 0.03 \mathrm{meV}$ and $q \simeq-17$ in agreement with [35].

When the resonance decays into several continuous channels, the additional correlation parameter $\rho$ needs to be introduced into the Fano cross-section formula [36]

$$
\sigma(\epsilon)=\sigma_{0}\left[1-\rho^{2}+\rho^{2} \frac{(q+\epsilon)^{2}}{1+\epsilon^{2}}\right] .
$$

In the simplest case of one dominant channel $\rho \lesssim 1$ [37],

$$
f(\epsilon)=f_{0}(\epsilon)\left[\zeta+\frac{q+\epsilon}{i+\epsilon}\right], \quad \zeta=\frac{\sqrt{1-\rho^{2}}}{\rho} .
$$

This modification allows both for the positive and negative WTD. Heuristically, this can be understood as a competition of "slow" and "fast" ionization channels, while switching from a slow to a fast channel may cause an advancement of the photoelectron. The peak value at the center of the resonance
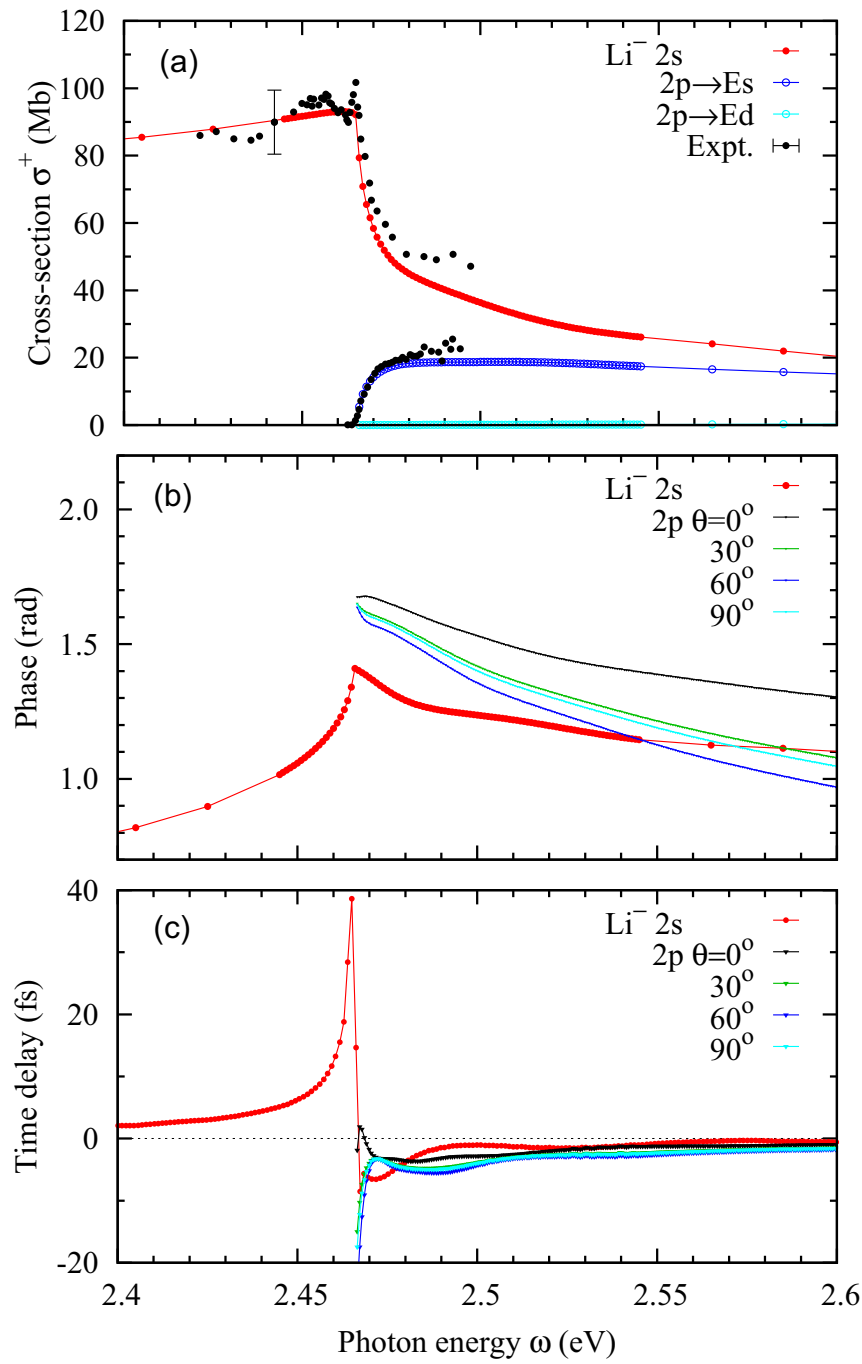

FIG. 2. Same as Fig. 1 for the $\mathrm{Li}^{-}$ion near the $2 p$ excitation threshold. Experimental cross-section results $[38,39]$ are displayed in the top panel.

becomes

$$
\tau_{0}=\frac{2}{\Gamma} \frac{1-\zeta / q^{2}}{1+\zeta^{2} / q^{2}} .
$$

This case applies to the broad resonance above the $n=2$ threshold centered at $10.97 \mathrm{eV}$. The peak time delay evaluated from the width $\Gamma=0.018 \mathrm{eV}$ amounts to $73 \mathrm{fs}$. The actual WTD in the $1 s \rightarrow E p$ channel is smaller, which can be understood qualitatively from Eq. (9). We see that all four autodetachment channels have very similar cross sections and Eq. (9) can only be used as an indication. The WTD in the $n=2$ channels is strongly angular dependent. Near the opening of the $n=2$ channels, it goes to very large negative values.

Figure 2 displays the set of $\mathrm{Li}^{-}$results analogous to that of $\mathrm{H}^{-}$shown in Fig. 1. The photodetachment cross section exhibited in the top panel of Fig. 2 displays a very clear threshold cusp prescribed by the Wigner threshold law $[2,40]$, which suppresses all the partial waves in the photoelectron wave packet in the newly opened channel except the $s$ wave. 
In the Wigner theory, the cross section near the opening of a new channel can be parametrized as

$$
\sigma(E)=\sigma_{0}-2 A\left|E-E_{0}\right|^{1 / 2}\left\{\begin{array}{lll}
\sin ^{2} \delta_{0} & \text { for } \quad E>E_{0} \\
\sin \delta_{0} \cos \delta_{0} & \text { for } & E<E_{0}
\end{array}\right.
$$

Equation (10) can be found in most quantum collision theory textbooks, i.e., Refs. [41-43]. A similar expression can be derived for the phase of the transition amplitude [44]:

$$
\delta(E)=\left\{\begin{array}{lll}
\delta_{0}-\kappa \chi^{2} \sin ^{2} \delta_{0} & \text { for } \quad E<E_{0}, \\
\delta_{0}+k \chi^{2} \sin \delta_{0} \cos \delta_{0} & \text { for } \quad E>E_{0} .
\end{array}\right.
$$

Here $k$ and $\kappa$ are defined by $k^{2}=\kappa^{2}=2\left(E-E_{0}\right)$ and $A=\chi^{2}$ is a coupling constant with the newly opened inelastic channel. The presence of a virtual bound state with the energy $E_{0}-\beta^{2} / 2$ changes this definition to $\kappa \rightarrow \beta \kappa /(\kappa+\beta)$ and $k \rightarrow \beta^{2} \kappa /\left(k^{2}+\beta^{2}\right)$ without changing the threshold behavior of the cross section (10) and the phase (11). The $\kappa$ or $k$ derivative of the phase (11) can become discontinuous. While this derivative is always negative below the threshold, its sign above the threshold is determined by the factor $\sin \delta_{0} \cos \delta_{0}$. In the case of $\mathrm{Li}^{-}, \delta_{0}$ is large and the factor is negative which explains the cusp exhibited in the top and middle panels of Fig. 2. Because of the Wigner cusp, the WTD at the $2 p$ threshold becomes divergent, which explains its sharp rise shown in the bottom panel. In the case of $\mathrm{H}^{-}$, which is illustrated in Fig. 1, the threshold phase is very close to zero and both the phase and the WTD remain continuous at the $n=2$ threshold. In comparison to $\mathrm{H}^{-}$, the angular dependence of the phase and WTD is rather weak in $\mathrm{Li}^{-}$. This is because the competing $2 p \rightarrow E d$ channel is strongly suppressed near the threshold and only becomes contributing away from it.

The stark difference between the $\mathrm{Li}^{-}$and $\mathrm{H}^{-}$threshold behavior is illustrated in Fig. 3. The top panel of this figure displays the angular anisotropy $\beta$ parameter in the $2 p$ channel. The general expression for the $\beta_{2 p}$ parameter [47] reduces to 0 or 2 in the limiting cases of the strong dominance of the $2 p \rightarrow E s$ or $2 p \rightarrow E d$ channels, respectively. The Wigner threshold law demands the strong suppression of the $2 p \rightarrow$ $E d$ channel and $\beta_{2 p} \propto-\left(E-E_{0}\right)$ above the threshold [48]. It is this behavior that is seen in $\mathrm{Li}^{-}$. In a sharp contrast, $\beta_{2 p} \simeq-1$ for $\mathrm{H}^{-}$, which indicates a comparable strength of the two channels. The only exception is the immediate vicinity of the threshold below $3 \mathrm{meV}$ excess energy. Here indeed $\beta_{2 p} \propto-\left(E-E_{0}\right)$, thus indicating the dominance of the $2 p \rightarrow E s$ photodetachment channel as required by the Wigner threshold law. Much further away from the threshold, the $2 p \rightarrow E d$ channel gradually takes over and $\beta \rightarrow 2$ in both ions while showing a rapid oscillation near the $n=3$ threshold. The higher thresholds are not resolved in the present calculation.

This threshold behavior is further highlighted in the middle and bottom panels of Fig. 3, which display the partial photodetachment cross sections in both ions plotted versus the absolute excess energy $\left|E-E_{0}\right|$. Below the threshold, the cross sections for the main photodetachment channel are folded over and overplotted versus $E_{0}-E$. The change of the slope of the two branches $E-E_{0}>0$ and $E-E_{0}<0$ indicates the presence of the cusp. This cusp is clearly visible in $\mathrm{Li}^{-}$and hardly noticeable in $\mathrm{H}^{-}$. The above analysis indicates
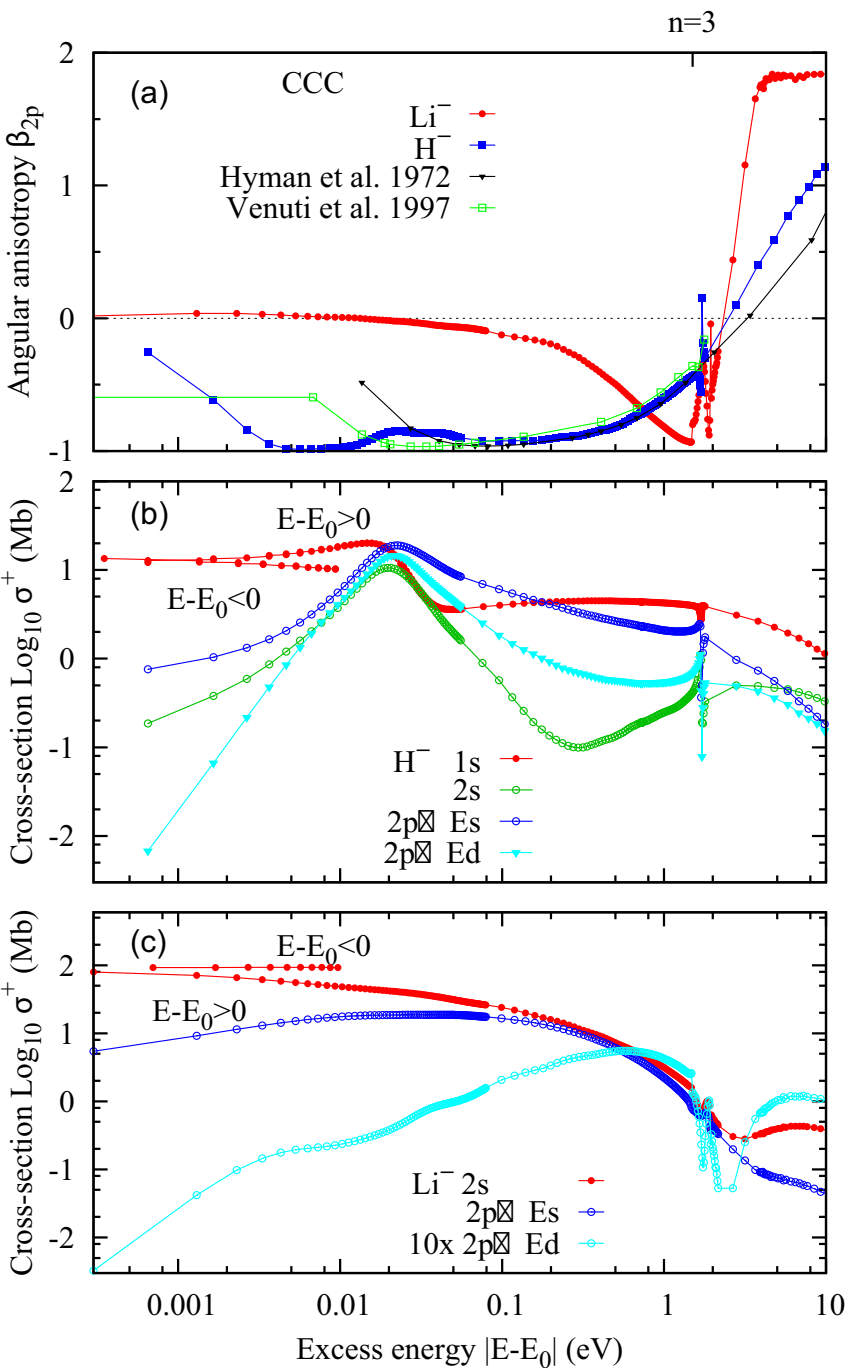

FIG. 3. (a) $\beta_{2 p}$ parameter in $\mathrm{Li}^{-}$and $\mathrm{H}^{-}$above the first excitation threshold plotted versus the excess energy. The $\mathrm{H}^{-}$data from Hyman et al. [45] and Venuti and Decleva [46] are also shown. The partial photoionization cross sections of $\mathrm{H}^{-}$(b) and $\mathrm{Li}^{-}$(c) are plotted on the absolute excess energy scale. Below threshold cross sections for the main ionization channel $1 s \rightarrow E p$ for $\mathrm{H}^{-}$and $2 s \rightarrow$ $E p$ for $\mathrm{Li}^{-}$are overplotted.

that the Wigner threshold law holds in both $\mathrm{H}^{-}$and $\mathrm{Li}^{-}$ions albeit on a very different excess energy scale. It is the presence of the virtual bound state in $\mathrm{Li}^{-}$that extends the validity of this law by at least two orders of magnitude. Similar behavior was detected in photodetachment of heavier alkali ions, $\mathrm{Na}^{-}$ and $\mathrm{K}^{-}$[49]. While $\mathrm{Li}^{-}$and $\mathrm{Na}^{-}$display very strong Wigner cusps, they are significantly reduced and narrowed in $\mathrm{K}^{-}$.

\section{B. Double photodetachment}

Now we address the second topic of the present investigation - the two-electron photodetachment with both electrons appearing in the continuum. In a general case, the timing analysis of such a process is complicated and depends sensitively on the photoelectron energy sharing and escape geometry [50]. This analysis is greatly simplified and 

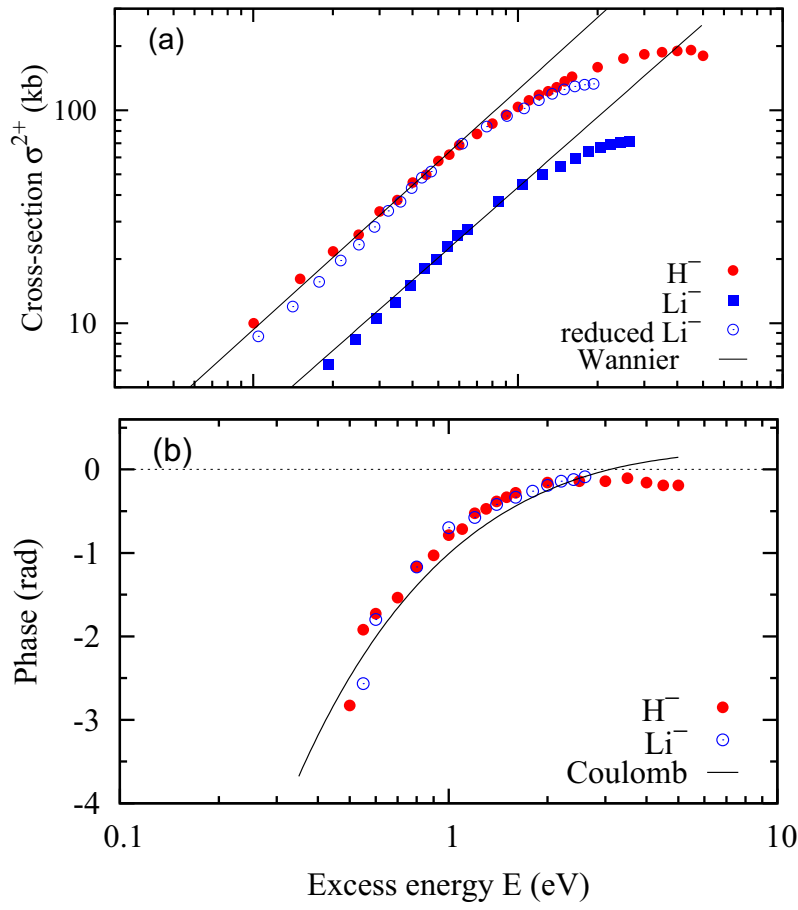

FIG. 4. (a) Double ionization cross sections of $\mathrm{H}^{-}$and $\mathrm{Li}^{-}$are plotted against the excess energy. The Wannier threshold law (12) is shown with the straight lines for both targets. The $\mathrm{Li}^{-}$cross section is also shown in the reduced coordinates $\sigma^{2+} Z^{4}$ vs $E / Z^{2}$, where $Z$ is the charge of the core. (b) Phase of the two-electron photodetachment amplitude (13) fitted with the $s$-wave Coulomb ansatz.

streamlined near the threshold of the two-electron escape. Near the threshold, the cross section follows the Wannier law [3]

$$
\sigma^{2+}(E, Z) \approx \sigma_{0}(Z) E^{M(Z)}
$$

where the exponent $M$ takes the values of 1.127 and 1.096 for $\mathrm{H}^{-}$and $\mathrm{Li}^{-}$, respectively. The effective core charge $Z_{\mathrm{Li}}=$ 1.13 for $\mathrm{Li}^{-}$is estimated by fitting the hydrogenic energy formula to the Rydberg series $\epsilon_{n s}=Z^{2} /\left(2 n^{2}\right)$. The calculated double ionization cross sections of $\mathrm{H}^{-}$and $\mathrm{Li}^{-}$satisfy this law very well as demonstrated in the top panel of Fig. 4. In this figure, we apply an additional scaling to the $\mathrm{Li}^{-}$cross section by reducing the $(y, x)$ coordinates to $\sigma^{2+} Z^{4}$ versus $E / Z^{2}$. This scaling brings both targets to a universal curve [24].

The Wannier threshold regime corresponds to the two continuum electrons receding back to back with equal energies, thus maintaining the electrostatic equilibrium of the system and avoiding falling back on the nucleus. Under these conditions, the two-electron detachment/ionization amplitude can be factorized into the dynamic and kinematic parts [51]:

$$
\left\langle\boldsymbol{k}_{1} \boldsymbol{k}_{2}|\mathcal{D}| \Psi_{0}\right\rangle=\mathcal{M}^{+}\left(E_{1}, E_{2}, \theta_{12}\right)\left[\hat{\boldsymbol{k}}_{1} \cdot \boldsymbol{e}+\hat{\boldsymbol{k}}_{2} \cdot \boldsymbol{e}\right] .
$$

Here the complex dynamic factor $\mathcal{M}^{+}\left(E_{1}, E_{2}, \theta_{12}\right)$ contains the energies of the photoelectrons and their mutual angle, while the kinematic factor depends on the photoelectrons' escape directions $\hat{\boldsymbol{k}}_{1}, \hat{\boldsymbol{k}}_{2}$ relative to the polarization axis of light $\boldsymbol{e}$. In the Wannier regime, $E_{1}=E_{2}$ and the amplitude $\mathcal{M}^{+}$peaks strongly at $\theta_{12}=\pi$. Because the kinematic factor is real, the phase of the amplitude (13) comes solely from the dynamic $\mathcal{M}^{+}$factor at the equal energy sharing and the backto-back emission. This phase in two-electron photodetachment of $\mathrm{H}^{-}$and $\mathrm{Li}^{-}$is plotted in the bottom panel of Fig. 4. It is fitted with the Coulomb $s$-wave ansatz given by Eq. (4) of [52]. This fit indicates that the two-electron photodetachment phase is divergent as $\eta \ln |\eta|$, where $\eta=-Z^{*} / k$ is the Sommerfeld parameter and $Z^{*} \simeq 0.55$. The WTD $\tau(E) \propto$ $E^{-3 / 2} \ln E$ becomes similarly divergent. A Coulomb $s$-wave behavior of the slow photoelectrons reaching the detector with an effective charge $Z^{*}<1$ can be understood by their mutual screening of the positively charged core $Z \simeq 1$. The departure of the two-electron phase from the Coulomb $s$ wave indicates noticeable contribution of other partial waves at the higher end of the energies considered in Fig. 4. The CCC model remains valid within a much wider excess energy range.

In conclusion, we demonstrate the threshold behavior of the Wigner time delay in photodetachment of negative atomic ions with two active electrons. We consider the $\mathrm{H}^{-}$and $\mathrm{Li}^{-}$ ions as the most representative and informative examples. The photoelectron phase in the elastic scattering channel near the lowest excitation threshold behaves very differently in these two ions. It is either small and continuous as in $\mathrm{H}^{-}$or large and cusplike as in $\mathrm{Li}^{-}$. Such a behavior is explained within the Wigner threshold theory $[2,40]$ modified by the presence of a virtual bound state [53]. As a result, the WTD, calculated as the energy derivative of the phase, remains finite as in $\mathrm{H}^{-}$or becomes divergent as in $\mathrm{Li}^{-}$. The $\mathrm{H}^{-}$ion demonstrates a sharp angular dependence of the WTD, whereas this dependence is rather weak in $\mathrm{Li}^{-}$. The WTD is divergent at the two-electron photodetachment threshold in both targets. Even though a large number of partial waves compose the two-electron wave packet, the slow electron reaches the detector as an $s$ wave propagating in an effective nucleus charge. The corresponding WTD displays a Coulomb singularity at the threshold. Such a behavior follows from the Wannier threshold theory [3] and it is common for all double ionization processes in both neutral and charged targets.

\section{SUMMARY AND OUTLOOK}

This work offers additional avenues for attosecond science to test atomic physics at the very fundamental level. The Wigner and Wannier threshold laws have been tested so far in the energy domain, while we suggest the way of their experimental verification in the time domain. Experimental observation of narrow resonant features in the threshold photodetachment WTD may seem beyond the present instrumental capabilities. However, the energy resolution attainable in attosecond experiments is rapidly improving. A resolution around $10 \mathrm{meV}$ is achieved in a RABBIT experiment [54] and very narrow resonant features become progressively resolved $[14,15,55,56]$.

Laser driven interferometric techniques [11,12] are relative in their nature. Observation of the angular dependent WTD facilitates such a relative measurement with reference to the polarization direction of light [57]. Two-electron escape in double ionization presents another opportunity with the WTD of the two photoelectrons being referenced against each other. While we consider here the equal energy sharing 
case, the presently developed theory allows one to interpret the two-electron escape with arbitrary energies by analyzing the phases of both the symmetric and antisymmetric amplitudes [58].

The WTD measurement in negative ions would be hampered by much lower ionic target densities as compared to neutral atomic targets. As a trade-off, the negative ions display gigantic time delays near their thresholds, of the order of femtoseconds. This should be much easier to detect in comparison to small, attosecond scale delays in neutral atoms. In laser driven atomic measurements, an additional correction due to the coupling of the laser field with the Coulomb field of the parent ion (CLC) needs to be introduced $[59,60]$. In resonant laser assisted measurements, the CLC correction may not fully decouple from the WTD $[61,62]$ and it becomes divergent near the threshold [63-65]. This correction remains finite and relatively small, of the order of $\sim 50$ as, in photodetachment of negative ions [66]. Therefore, it will be hardly noticeable in photodetachment near the excitation threshold. In double detachment, the Coulomb field of the core does couple with the laser field. However, both the CLC correction and the WTD display the same Coulomb singularity of the opposite sign. As a result, the measured phase flattens near the threshold $[67,68]$. In the present case, the Coulomb phase of the slow photoelectron carries an imprint of an effective charge $Z^{*}$, which differs from the charge of the core $Z$. So the interplay of the Wigner phase and the CLC correction may be particularly interesting.

The present theoretical findings are based solely on the Wigner and Wannier threshold laws and do not depend on the specific numerical implementation. Once confirmed experimentally, these results will establish the fundamental threshold laws in the time-resolved atomic collision physics. Otherwise, the laws of nature will be revealed in the threshold collision physics studied in the time domain.

\section{ACKNOWLEDGMENTS}

We acknowledge the use of resources of the National Computational Infrastructure facility (NCI Australia).
[1] H. R. Sadeghpour, J. L. Bohn, M. J. Cavagnero, B. D. Esry, I. I. Fabrikant, J. H. Macek, and A. R. P. Rau, Collisions near threshold in atomic and molecular physics, J. Phys. B 33, R93 (2000).

[2] E. P. Wigner, On the behavior of cross sections near thresholds, Phys. Rev. 73, 1002 (1948).

[3] G. H. Wannier, The threshold law for single ionization of atoms or ions by electrons, Phys. Rev. 90, 817 (1953).

[4] M. T. Eiles and C. H. Greene, Extreme Correlation and Repulsive Interactions in Highly Excited Atomic Alkali Anions, Phys. Rev. Lett. 121, 133401 (2018).

[5] T. J. Babij, J. R. Machacek, D. J. Murtagh, S. J. Buckman, and J. P. Sullivan, Near-Threshold Ionization of Argon by Positron Impact, Phys. Rev. Lett. 120, 113401 (2018).

[6] I. Bray, A. W. Bray, D. V. Fursa, and A. S. Kadyrov, NearThreshold Cross Sections for Electron and Positron Impact Ionization of Atomic Hydrogen, Phys. Rev. Lett. 121, 203401 (2018).

[7] R. Pazourek, S. Nagele, and J. Burgdörfer, Attosecond chronoscopy of photoemission, Rev. Mod. Phys. 87, 765 (2015).

[8] L. Eisenbud, Formal properties of nuclear collisions, Ph.D. thesis, Princeton University, 1948.

[9] E. P. Wigner, Lower limit for the energy derivative of the scattering phase shift, Phys. Rev. 98, 145 (1955).

[10] F. T. Smith, Lifetime matrix in collision theory, Phys. Rev. 118, 349 (1960)

[11] M. Schultze, M. Fiess, N. Karpowicz, J. Gagnon, M. Korbman, M. Hofstetter, S. Neppl, A. L. Cavalieri, Y. Komninos, T. Mercouris et al., Delay in photoemission, Science 328, 1658 (2010).

[12] K. Klünder, J. M. Dahlström, M. Gisselbrecht, T. Fordell, M. Swoboda, D. Guénot, P. Johnsson, J. Caillat, J. Mauritsson, A. Maquet, R. Taïeb, and A. L'Huillier, Probing Single-Photon Ionization on the Attosecond Time Scale, Phys. Rev. Lett. 106, 143002 (2011).
[13] S. Haessler, B. Fabre, J. Higuet, J. Caillat, T. Ruchon, P. Breger, B. Carré, E. Constant, A. Maquet, E. Mével, P. Salières, R. Taïeb, and Y. Mairesse, Phase-resolved attosecond nearthreshold photoionization of molecular nitrogen, Phys. Rev. A 80, 011404(R) (2009).

[14] C. Cirelli, C. Marante, S. Heuser, C. L. M. Petersson, A. J. Galán, L. Argenti, S. Zhong, D. Busto, M. Isinger, S. Nandi et al., Anisotropic photoemission time delays close to a Fano resonance, Nat. Commun. 9, 955 (2018).

[15] S. Nandi, E. Plésiat, S. Zhong, A. Palacios, D. Busto, M. Isinger, L. Neoričić, C. L. Arnold, R. J. Squibb, R. Feifel et al., Attosecond timing of electron emission from a molecular shape resonance, Sci. Adv. 6, eaba7762 (2020).

[16] A. Kamalov, A. L. Wang, P. H. Bucksbaum, D. J. Haxton, and J. P. Cryan, Electron correlation effects in attosecond photoionization of $\mathrm{CO}_{2}$, Phys. Rev. A 102, 023118 (2020).

[17] A. S. Kheifets, A. W. Bray, and I. Bray, Attosecond Time Delay in Photoemission and Electron Scattering Near Threshold, Phys. Rev. Lett. 117, 143202 (2016).

[18] S. J. Buckman and C. W. Clark, Atomic negative-ion resonances, Rev. Mod. Phys. 66, 539 (1994).

[19] V. K. Ivanov, Many-body effects in negative ion photodetachment, J. Phys. B 32, R67 (1999).

[20] V. Ivanov, Theoretical studies of photodetachment, Radiat. Phys. Chem. 70, 345 (2004).

[21] E. Lindroth and J. Luis Sanz-Vicario, Photodetachment of few-electron negative ions, Radiat. Phys. Chem. 70, 387 (2004).

[22] D. J. Pegg, Structure and dynamics of negative ions, Rep. Prog. Phys. 67, 857 (2004).

[23] T. Andersen, Atomic negative ions: structure, dynamics and collisions, Phys. Rep. 394, 157 (2004).

[24] A. S. Kheifets and I. Bray, Photoionization with excitation and double photoionization of the helium isoelectronic sequence, Phys. Rev. A 58, 4501 (1998). 
[25] A. S. Kheifets and I. Bray, Valence-shell double photoionization of alkaline-earth-metal atoms, Phys. Rev. A 75, 042703 (2007).

[26] K. G. Dyall, I. P. Grant, C. T. Johnson, F. P. Parpia, and E. P. Plummer, GRASP: A general-purpose relativistic atomic structure program, Comput. Phys. Commun. 55, 425 (1989).

[27] A. Kramida, Yu. Ralchenko, J. Reader, and NIST ASD Team, NIST Atomic Spectra Database (ver. 5.2) (National Institute of Standards and Technology, Gaithersburg, MD, 2014), http: //physics.nist.gov/asd.

[28] K. R. Lykke, K. K. Murray, and W. C. Lineberger, Threshold photodetachment of $\mathrm{H}^{-}$, Phys. Rev. A 43, 6104 (1991).

[29] G. Haeffler, D. Hanstorp, I. Kiyan, A. E. Klinkmüller, U. Ljungblad, and D. J. Pegg, Electron affinity of Li: A stateselective measurement, Phys. Rev. A 53, 4127 (1996).

[30] M. Y. Amusia, Atomic Photoeffect (Plenum Press, New York, 1990).

[31] A. S. Kheifets and I. Bray, Calculation of double photoionization of helium using the convergent close-coupling method, Phys. Rev. A 54, R995(R) (1996).

[32] H. H. Andersen, P. Balling, P. Kristensen, U. V. Pedersen, S. A. Aseyev, V. V. Petrunin, and T. Andersen, Positions and Isotope Shifts of the $\mathrm{H}^{-}\left({ }^{1} P^{0}\right)$ Dipole Resonances Below the $\mathrm{H}(n=2)$ Threshold, Phys. Rev. Lett. 79, 4770 (1997).

[33] P. G. Harris, H. C. Bryant, A. H. Mohagheghi, R. A. Reeder, C. Y. Tang, J. B. Donahue, and C. R. Quick, Observation of doubly excited resonances in the $\mathrm{H}^{-}$ion, Phys. Rev. A 42, 6443 (1990).

[34] U. Fano, Effects of configuration interaction on intensities and phase shifts, Phys. Rev. 124, 1866 (1961).

[35] E. Lindroth, Photodetachment of $\mathrm{H}^{-}$and $\mathrm{Li}^{-}$, Phys. Rev. A 52, 2737 (1995)

[36] U. Fano and J. W. Cooper, Line profiles in the far-uv absorption spectra of the rare gases, Phys. Rev. 137, A1364 (1965).

[37] P. C. Deshmukh, A. Kumar, H. R. Varma, S. Banerjee, S. T. Manson, V. K. Dolmatov, and A. S. Kheifets, WignerEisenbud-Smith photoionization time delay due to autoioinization resonances, J. Phys. B 51, 065008 (2018).

[38] J. Dellwo, Y. Liu, D. J. Pegg, and G. D. Alton, Near-threshold photodetachment of the $\mathrm{Li}^{-}$ion, Phys. Rev. A 45, 1544 (1992).

[39] J. Dellwo, Y. Liu, C. Y. Tang, D. J. Pegg, and G. D. Alton, Photodetachment cross sections for $\mathrm{Li}^{-}$, Phys. Rev. A 46, 3924 (1992).

[40] A. Baz', Y. Zel'dovich, and A. Perelomov, Scattering, Reactions and Decay in Nonrelativistic Quantum Mechanics (Israel Program for Scientific Translation, Jerusalem, 1966).

[41] R. G. Newton, Scattering Theory of Waves and Particles, 2nd ed., Texts and Monographs in Physics (Springer-Verlag, New York, 1982).

[42] L. D. Landau and E. M. Lifshitz, Quantum Mechanics (Nonrelativistic theory), 3rd ed., Course of Theoretical Physics Vol. 3 (Pergamon Press, Oxford, 1985).

[43] N. Mott and H. Massey, Theory of Atomic Collisions (Oxford University Press, New York, 1987).

[44] R. K. Nesbet, Multichannel threshold structures in scattering theory, J. Phys. B 13, L193 (1980).

[45] H. A. Hyman, V. L. Jacobs, and P. G. Burke, Photoionization of $\mathrm{H}^{-}$and He above the $\mathrm{n}=2$ threshold, J. Phys. B 5, 2282 (1972).
[46] M. Venuti and P. Decleva, Convergent multichannel continuum states by a general configuration interaction expansion in a Bspline basis: application to $\mathrm{H}^{-}$photodetachment, J. Phys. B: At., Mol., Opt. Phys. 30, 4839 (1997).

[47] J. Cooper and R. N. Zare, Angular distribution of photoelectrons, J. Chem. Phys. 48, 942 (1968).

[48] M. Y. Amusia, A. S. Baltenkov, L. V. Chernysheva, Z. Felfli, and A. Z. Msezane, Near-threshold behavior of angular anisotropy parameters in negative-ion photodetachment, Phys. Rev. A 72, 032727 (2005).

[49] D. L. Moores and D. W. Norcross, Alkali-metal negative ions. I. Photodetachment of $\mathrm{Li}^{-}, \mathrm{Na}^{-}$, and $\mathrm{K}-$, Phys. Rev. A 10, 1646 (1974).

[50] A. S. Kheifets, I. A. Ivanov, and I. Bray, Timing analysis of two-electron photoemission, J. Phys. B 44, 101003 (2011).

[51] A. S. Kheifets and I. Bray, Equal energy-sharing double photoionization of helium from near-threshold to high energies, Phys. Rev. A 62, 065402 (2000).

[52] J. C. A. Barata, L. F. Canto, and M. S. Hussein, New asymptotic formulae for the point Coulomb phase shift, Braz. J. Phys. 41, 50 (2011).

[53] Y. K. Bae and J. R. Peterson, Effect of a virtual state near an s-wave threshold: Absolute Li photodetachment cross sections near the Li ( $\left.2^{2} \mathrm{p}\right)$ threshold, Phys. Rev. A 32, 1917 (1985).

[54] K. T. Kim, D. H. Ko, J. Park, V. Tosa, and C. H. Nam, Complete temporal reconstruction of attosecond high-harmonic pulse trains, New J. Phys. 12, 083019 (2010).

[55] V. Gruson, L. Barreau, Á. Jiménez-Galan, F. Risoud, J. Caillat, A. Maquet, B. Carré, F. Lepetit, J.-F. Hergott, T. Ruchon et al., Attosecond dynamics through a Fano resonance: Monitoring the birth of a photoelectron, Science 354, 734 (2016).

[56] M. Kotur, D. Guénot, A. Jiménez-Galán, D. Kroon, E. W. Larsen, M. Louisy, S. Bengtsson, M. Miranda, J. Mauritsson, C. L. Arnold et al., Spectral phase measurement of a Fano resonance using tunable attosecond pulses, Nat. Commun. 7, 10566 (2016).

[57] S. Heuser, A. Jiménez Galán, C. Cirelli, C. Marante, M. Sabbar, R. Boge, M. Lucchini, L. Gallmann, I. Ivanov, A. S. Kheifets, J. M. Dahlstrom, E. Lindroth, L. Argenti, F. Martin, and U. Keller, Angular dependence of photoemission time delay in helium, Phys. Rev. A 94, 063409 (2016).

[58] A. S. Kheifets and I. Bray, Symmetrized amplitudes of the helium-atom double photoionization, Phys. Rev. A 65, 022708 (2002).

[59] J. Dahlström, D. Guénot, K. Klünder, M. Gisselbrecht, J. Mauritsson, A. L. Huillier, A. Maquet, and R. Taïeb, Theory of attosecond delays in laser-assisted photoionization, Chem. Phys. 414, 53 (2012).

[60] S. Nagele, R. Pazourek, J. Feist, and J. Burgdörfer, Time shifts in photoemission from a fully correlated two-electron model system, Phys. Rev. A 85, 033401 (2012).

[61] L. Argenti, A. Jiménez-Galán, J. Caillat, R. Taïeb, A. Maquet, and F. Martín, Control of photoemission delay in resonant twophoton transitions, Phys. Rev. A 95, 043426 (2017).

[62] S. Beaulieu, A. Comby, A. Clergerie, J. Caillat, D. Descamps, N. Dudovich, B. Fabre, R. Géneaux, F. Légaré, S. Petit et al., Attosecond-resolved photoionization of chiral molecules, Science 358, 1288 (2017).

[63] M. Ivanov and O. Smirnova, How Accurate is the Attosecond Streak Camera?, Phys. Rev. Lett. 107, 213605 (2011). 
[64] R. Pazourek, S. Nagele, and J. Burgdörfer, Time-resolved photoemission on the attosecond scale: Opportunities and challenges, Faraday Discuss. 163, 353 (2013).

[65] V. V. Serov, V. L. Derbov, and T. A. Sergeeva, Interpretation of time delay in the ionization of two-center systems, Phys. Rev. A 87, 063414 (2013).

[66] E. Lindroth and J. M. Dahlström, Attosecond delays in laserassisted photodetachment from closed-shell negative ions, Phys. Rev. A 96, 013420 (2017).
[67] G. Schmid, K. Schnorr, S. Augustin, S. Meister, H. Lindenblatt, F. Trost, Y. Liu, N. Stojanovic, A. Al-Shemmary, T. Golz, R. Treusch, M. Gensch, M. Kubel, L. Foucar, A. Rudenko, J. Ullrich, C. D. Schroter, T. Pfeifer, and R. Moshammer, Terahertz-Field-Induced Time Shifts in Atomic Photoemission, Phys. Rev. Lett. 122, 073001 (2019).

[68] A. S. Kheifets and A. W. Bray, RABBITT phase transition across the ionization threshold, Phys. Rev. A 103, L011101 (2021). 